'Servicio Ginecología y Obstetricia Hospital Parroquial San Bernardo. Santiago, Chile. 2Facultad de Medicina, Universidad de los Andes. Santiago, Chile. ${ }^{3}$ Departamento Salud Pública y Epidemiología. Santiago, Chile. IInterno de Medicina,, Facultad de Medicina, Universidad de los Andes. Santiago, Chile.

Recibido el 10 de diciembre de 2015, aceptado el 17 de junio de 2016.

Correspondencia a: Cinthya Urquid curquidi@uandes.cl

\section{Sensibilidad al cambio de la versión español-chileno del cuestionario International Consultation on Incontinence Questionnaire Short-Form (ICIQ-SF) para incontinencia urinaria femenina}

\author{
MARITZA BUSQUETS ${ }^{1,2}$, CINTHYA URQUIDI $^{2,3}$, EMILIANO PERTOSSI $^{2, \mathrm{a}}$
}

\begin{abstract}
Background: A symptom questionnaire must be able to detect minimal changes after treatment to be clinically useful. Aim: To evaluate the responsiveness of the Spanish-Chilean version of the ICIQ-SF questionnaire after medical and surgical procedures for the treatment of urinary incontinence (UI) in women consulting at a public Chilean Hospital. Material and Methods: Data of 124 women aged $59 \pm 10$ years who were treated for UI and in whom ICIQ-SF questionnaire was applied in two opportunities (before and after medical or surgical treatment), was analyzed. The sign test for paired samples was used to compare the response of each item of the questionnaire. Responsiveness of the total severity score for UI was assessed by changes of slopes estimated by Generalized Estimating Equations. Slopes of change for every type of treatment were also compared. Results: Sixty two percent of the women underwent surgical treatment. The slope of the total severity score for medical treatment was -4.4 ( $p$-value $<0.001$ for change) and 12.6 ( $p$-value $<0.001$ for change) for surgical treatment. Changes in surgical treatment were significantly higher than those of medical treatment ( $p$-value $<0.001)$. Conclusions: The Spanish-Chilean version of the ICIQ-SF questionnaire was able to detect changes as a result of surgical and medical treatment for urinary incontinence in the study population, fulfilling the validity of responsiveness criterion.
\end{abstract}

(Rev Med Chile 2016; 144: 1006-1011)

Key words: Quality of Life; Reproducibility of Results; Surveys and Questionnaires; Urinary Incontinence.
L a incontinencia urinaria (IU) femenina, definida como cualquier emisión involuntaria de orina, es un problema de salud importante en la mujer. En la población general su prevalencia es del orden de $30 \%$ y hasta $50 \%$ en mujeres sobre los 60 años ${ }^{1}$.
Clínicamente la IU se clasifica como de esfuerzo, urgencia y/o mixta. Desde el punto de vista fisiopatológico, en la IU de esfuerzo (que se presenta con la tos, estornudo o ejercicio físico), hay una falla anatómica del mecanismo de cierre uretro-vesical que es factible de corrección 
quirúrgica. En el caso de IU de urgencia (que se presenta luego del deseo imperioso de micción), existe una contracción del músculo detrusor que no puede ser inhibida desde centros superiores y corresponde a lo que se denomina detrusor hiperactivo. El detrusor hiperactivo da lugar, clínicamente, a la vejiga hiperactiva, que puede cursar con o sin incontinencia de orina y su manejo es principalmente médico. Se clasifica como IU mixta cuando ambos componentes están presentes en una proporción que varía según el grupo etario ${ }^{2}$. El manejo comenzará por el tipo de IU que la mujer refiere como el más molesto o prevalente ${ }^{3}$. El tratamiento quirúrgico consiste principalmente en la colocación de mallas suburetrales libres de tensión, TVT ("trans vaginal free tape") y TOT ("trans obturator free vaginal tape") ${ }^{4}$. El manejo médico consta de múltiples opciones con distintos grados de recomendación ${ }^{3}$. Actualmente el manejo conductual es de primera línea (reeducación vesical, manejo de la ingesta de líquidos, reforzamiento muscular de piso pelviano y otros) y de segunda línea el uso de anticolinérgicos ${ }^{5,6}$. El uso de terapia estrogénica local se utiliza en casos de atrofia genitourinaria en mujeres posmenopáusicas ${ }^{7}$.

Además de la valoración clínica efectuada por el médico, la percepción del propio paciente respecto al cambio en sus síntomas y calidad de vida se considera indispensable al momento de evaluar una conducta terapéutica. Por ello, se han establecido guías para la elaboración y valoración de instrumentos de resultados percibidos por los propios pacientes ${ }^{8}$. Entre los diversos instrumentos para la evaluación de la IU femenina, el ICIQSF (International Consultation on Incontinence Questionnaire Short-Form $)^{9}$ es un cuestionario breve dirigido a medir la percepción de los síntomas de IU y la calidad de vida de la paciente. Previamente, este cuestionario ha sido validado en una población chilena usuaria del Sistema Público de Salud cumpliendo adecuadamente con los criterios de validez de contenido, constructo y de criterio; como así también con la precisión y reproducibilidad ${ }^{10}$, obteniendo como resultado una versión español-chileno. Sin embargo, la utilidad de un instrumento no solamente depende de su validez y confiabilidad sino también que debe ser capaz de detectar cambios mínimos, pero clínicamente relevantes en el tiempo; esta propiedad se denomina sensibilidad al cambio ${ }^{11}$.
En este sentido, en un contexto donde los cambios clínicamente relevantes que se atribuyen a una determinada conducta terapéutica, debieran ser percibidos por el instrumento.

Por lo tanto, el propósito de este estudio es concluir con el proceso de validación de la versión español-chileno del cuestionario ICIQ-SF en una población de mujeres chilenas, evaluando la sensibilidad al cambio atribuible al tratamiento de la IU. De esta forma, este cuestionario podrá ser utilizado en la rutina del manejo de las pacientes y en el contexto de la investigación clínica o epidemiológica.

\section{Pacientes y Métodos}

Se llevó a cabo un estudio de cohorte histórica en base a los registros de 124 mujeres con diagnóstico clínico de IU entre los años 2005 y 2006. En ellas, se había administrado el cuestionario ICIQSF en su versión español-chileno, antes y entre 2 a 12 semanas después de la conducta terapéutica. El estudio y el uso de esta información fueron aprobados por Comité de Ética del Hospital Parroquial San Bernardo. Para asegurar la confidencialidad de las participantes, se construyó una base de datos con un código de identificación que no incluyó el nombre $\mathrm{u}$ otros antecedentes relevantes que pudiesen identificar al sujeto.

La población de estudio fueron mujeres beneficiarias del Fondo Nacional de Salud (FONASA) del Servicio de Uroginecología del Hospital Parroquial de San Bernardo (HPSB) de la Región Metropolitana, quienes se habían sometido a tratamiento quirúrgico o manejo médico para IU. Los detalles del reclutamiento y la autorización del uso del cuestionario se detallan en una publicación previa ${ }^{10}$.

El manejo médico de la IU se efectuó en base a las posibilidades del Servicio ya que en el período descrito no se contaba con kinesiólogos especialistas en piso pelviano, ni tampoco con posibilidades de manejo conductual por parte de personal calificado; en este contexto, se utilizó terapia hormonal, anticolinérgicos o una combinación de ambos. El tratamiento quirúrgico que se realizó fue la colocación de malla sub-uretral libre de tensión transobturador (TOT), ya que ha sido la técnica adoptada por la mayoría de los ginecólogos del Servicio. La elección del tipo de 
tratamiento se realizó en base al predominio de las molestias derivadas de la IU de urgencia o de esfuerzo, referidas por la paciente y en los casos en que no había una clara prevalencia, se efectuó TOT en primera instancia sólo en las pacientes portadoras de prolapso sintomático de resorte también quirúrgico.

La versión chileno-español del cuestionario ICIQ-SF forma parte de la evaluación clínica de rutina de la Unidad de Uroginecología del HPSB y es facilitado por el personal médico para que pueda ser auto-administrado por las pacientes. El cuestionario, en su versión español-chileno ${ }^{10}$ está compuesto por 4 ítems en total, dónde los tres primeros evalúan el grado de severidad de la incontinencia a través de un puntaje en escala ordinal. Dado que el último ítem aborda el tipo de situaciones en que la paciente pierde orina, éste fue excluido del análisis. Por lo tanto, los ítems analizados fueron: 1) frecuencia de pérdida de orina; 2) cantidad de pérdida de orina y 3 ) afectación de calidad de vida. Con la suma de los puntajes de cada ítem se obtiene el puntaje total de severidad, donde el mínimo de 0 puntos representa el menor grado de severidad y 21 el mayor grado.

De la misma fuente se usó la información de: tipo de tratamiento (médico o quirúrgico), edad (años cumplidos) e Índice de Masa Corporal (IMC), calculado como peso en $\mathrm{kg} /$ talla en $\mathrm{m}^{2}$.

Las variables continuas se resumieron con promedios y desviaciones estándar (DE) dado la simetría de su distribución, y las ordinales con medianas y rangos intercuartílicos (RIC). Para la comparación de cada ítem por tipo de tratamiento y pre-post tratamiento, se usó la prueba de Wilcoxon para muestras independientes y la de Signos para muestras pareadas respectivamente. La sensibilidad al cambio fue evaluada a través del ajuste de modelos semi-paramétricos de Ecuaciones de Estimación Generalizada (EEG) de manera de controlar las diferencias basales entre sujetos y la correlación de las mediciones pre-post tratamiento. Al modelo se le incluyó un término de interacción entre el tipo de tratamiento y el tiempo de seguimiento para comparar las pendientes de cambios entre tipos de tratamientos. El criterio de decisión fue un p-value a dos colas menor a 0,05 y se reportan los coeficientes betas estimados por EEG. El análisis se hizo con el paquete estadístico Stata/SE 13.0 (Statcorp, Texas) con licencia para la Universidad de los Andes.

\section{Resultado}

Del total de la información disponible (124 mujeres), se excluyeron dos casos por no presentar registros del ICIQ-SF después del tratamiento. De un total de 122 observaciones, 76 casos $(62,3 \%)$ estuvieron bajo tratamiento médico para la IU y $46(37,7 \%)$ bajo el quirúrgico. Se dispuso registros completos de peso y talla en $65,5 \%$ de los casos.

En la Tabla 1, se detalla una descripción completa de la edad, la antropometría y las respuestas al cuestionario ICIQ-SF en el total de la población de estudio y la comparación por tipo de tratamiento (médico y quirúrgico). El promedio de edad fue de 59,1 años con desviación estándar (DE) de 10,1, ésta fue similar en las mujeres bajo tratamiento quirúrgico versus médico $(p-v a-$ $l u e=0,696$ ). El IMC promedio para todo el grupo fue de $30,4 \mathrm{~kg} / \mathrm{m}^{2}$ (DE: 5,3 ), las mujeres que se sometieron a tratamiento quirúrgico presentaron un mayor IMC que aquella bajo tratamiento médico $\left(31,8 \mathrm{~kg} /\right.$ talla $^{2}$ versus 29,2 respectivamente; $p$-value $=0,030)$. Con respecto a las respuestas del cuestionario ICIQ-SF antes del tratamiento, no se encontraron diferencias significativas en las mujeres bajo tratamiento médico versus quirúrgico ( $p$-value $>0,05)$; no obstante, la mediana de las respuestas después del tratamiento fue mayor en el grupo con tratamiento médico comparado con el quirúrgico ( $p$-value $<0,001)$ (Tabla 1).

En la Tabla 2 se muestra la proporción de mujeres que presentaron una evolución positiva después del tratamiento (médico o quirúrgico) detectado por el cambio en la severidad del puntaje de cada ítem del cuestionario ICIQ-SF. Los tres ítems del cuestionario, identificaron cambios significativos en el puntaje para ambos tipos de tratamiento; sin embargo, la proporción de mujeres con evolución positiva fue superior a $80 \%$ bajo el tratamiento quirúrgico; en contra parte, ésta no superó el 68,5\% en el tratamiento médico (Tabla 2).

En la Figura 1 se muestra la sensibilidad al cambio del puntaje total de severidad por cada tipo de tratamiento. La pendiente de cambio, estimado por el coeficiente beta, fue negativa y significativa para ambos tipos de tratamiento ( $p$-value $<0,001)$. El puntaje de severidad de IU de la versión chileno-español del ICIQ-SF, identificó una magnitud del cambio bajo el tratamiento quirúrgico, expresado por la pendiente (beta), significativamente mayor que el tratamiento médico ( $p$-value $<0,001)$. 
Tabla 1. Descripción de la población de estudio y comparación por tipo de tratamiento médico y quirúrgico

\begin{tabular}{|c|c|c|c|c|}
\hline & $\begin{array}{c}\text { Total } \\
n=122\end{array}$ & $\begin{array}{c}\text { Médico } \\
n=46\end{array}$ & $\begin{array}{c}\text { Quirúrgico } \\
n=76\end{array}$ & p-value $^{\dagger}$ \\
\hline Edad (años) & $59,1(10,1)$ & $59,0(11,0)$ & $59,1 \quad(8,3)$ & 0,696 \\
\hline Peso (kg) & $71,2(12,2)$ & $68,7(10,3)$ & $73,9(13,7)$ & 0,096 \\
\hline Talla (m) & $1,54(0,6)$ & $1,55(0,07)$ & $1,54(0,05)$ & 0,143 \\
\hline IMC $\left(\mathrm{kg} / \mathrm{m}^{2}\right)$ & $30,4(5,3)$ & $29,2(4,8)$ & $31,8 \quad(5,7)$ & 0,030 * \\
\hline $\begin{array}{l}\text { Ítem 1: Frecuencia de pérdida de orina } \\
\text { Antes } \\
\text { Después }\end{array}$ & $\begin{array}{ll}4 & (1) \\
2 & (4)\end{array}$ & $\begin{array}{ll}4 & (1) \\
3 & (3)\end{array}$ & $\begin{array}{ll}4 & (2) \\
0 & (2)\end{array}$ & $\begin{aligned} & 0,703 \\
< & 0,001^{*}\end{aligned}$ \\
\hline $\begin{array}{l}\text { Ítem 2: Frecuencia de pérdida de orina } \\
\text { Antes } \\
\text { Después }\end{array}$ & $\begin{array}{ll}4 & (2) \\
2 & (4)\end{array}$ & $\begin{array}{ll}4 & (0) \\
2 & (2)\end{array}$ & $\begin{array}{ll}4 & (2) \\
0 & (2)\end{array}$ & $\begin{aligned} & 0,143 \\
< & 0,001 *\end{aligned}$ \\
\hline $\begin{array}{l}\text { Ítem 3: Afectación de calidad de vida } \\
\text { Antes } \\
\text { Después }\end{array}$ & $\begin{array}{rr}10 & (4) \\
3 & (8)\end{array}$ & $\begin{array}{ll}9 & (4) \\
5 & (7)\end{array}$ & $\begin{array}{rr}10 & (4) \\
0 & (3)\end{array}$ & $\begin{array}{r}0,259 \\
<0,001 \text { * }\end{array}$ \\
\hline
\end{tabular}

La antropometría y la edad están expresado con promedios y desviación estándar entre (). Los ítems de la versión español-chileno del ICIQ-SF con medianas y rangos intercuartílicos entre (). IMC: Índice de Masa Corporal. †Prueba de Wilcoxon para muestras independientes, comparación de Médico versus Quirúrgico. *Estadísticamente significativo $(p$-value $<0,05)$.

Tabla 2. Proporción de mujeres con evolución positiva antes y después del tratamiento, identificado por la versión chileno-español del cuestionario ICIQ-SF

\begin{tabular}{|lccc|}
\hline ICIQ-SF & Total & Médico & Quirúrgico \\
& $\mathbf{n = 1 2 2}$ & $\mathbf{n = 7 6}$ & $\mathbf{n = 4 6}$ \\
\hline Ítem 1: Frecuencia de pérdida de orina & $68,6(83)^{*}$ & $68,5(46)^{*}$ & $88,9(40)^{*}$ \\
Ítem 2: Cantidad de pérdida de orina & $62,3(76)^{*}$ & $48,7(37)^{*}$ & $84,8(39)^{*}$ \\
Ítem 3: Afectación de calidad de vida & $71,3(82)^{*}$ & $57,9(44)^{*}$ & $93,5(43)^{*}$ \\
\hline
\end{tabular}

Todas las variables están expresadas en $\%$ y frecuencias entre (). ${ }^{*} p$-value significativo $(<0,001)$ de la diferencia de medianas antes y después del tratamiento. Prueba del signo para muestras pareadas.

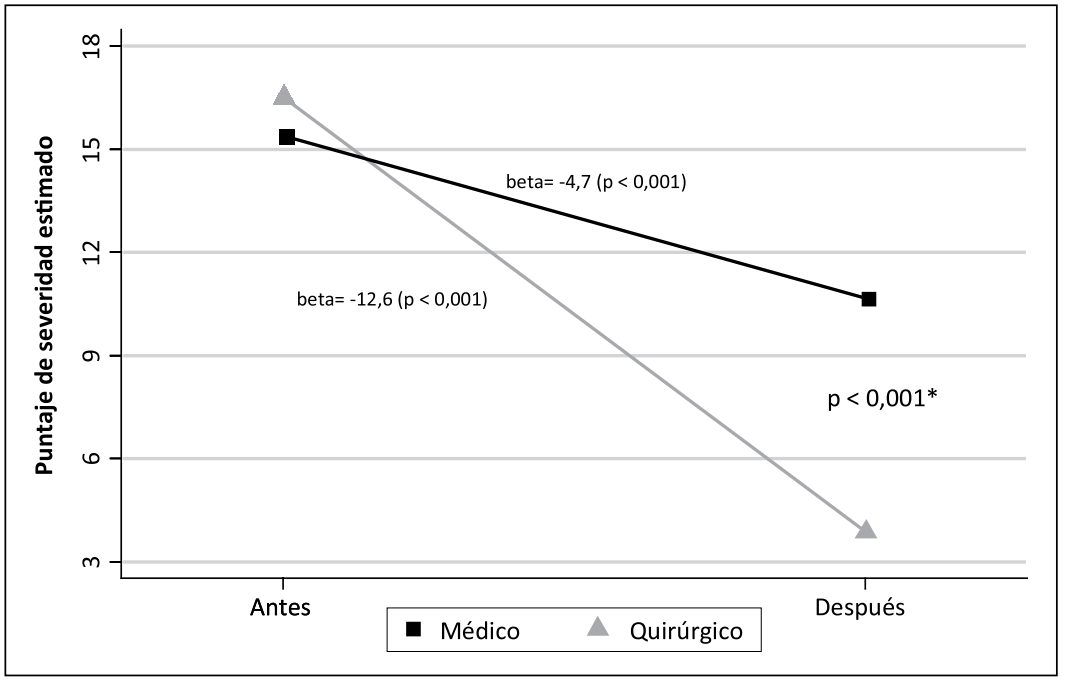

Figura 1. Sensibilidad al cambio del puntaje total de severidad de incontinencia urinaria, obtenido del cuestionario ICIQ-SF, antes y después del tratamiento y comparado según tipo de tratamiento. p: $p$-value del cambio post-tratamiento. $p^{*}: p$-value del cambio del tratamiento médico versus quirúrgico. Los resultados fueron similares después de ajustar por Índice de Masa Corporal. 


\section{Discusión}

Con los resultados de este estudio se ha culminado el proceso de validación de la versión español-chileno del cuestionario ICQ-SF (International Consultation on Incontinence Questionnaire Short-Form).

La calidad de vida corresponde uno de los desenlaces más relevantes a la hora de evaluar la efectividad de tratamientos para la incontinencia urinaria (IU); como así también para poder estimar el éxito y corregir conductas terapéuticas en una Unidad de Uroginecología; por lo tanto, es necesario contar con instrumentos validados localmente y sensibles para identificar cambios a lo largo del tiempo. Si bien el cuestionario Kings Health Questionnaire, traducido y validado adecuadamente al español ${ }^{12}$, podría cubrir estas necesidades, este fue ideado para evaluar disfunciones urinarias en general. En cambio, el ICIQ-SF fue diseñado y validado específicamente para síntomas de IU y su impacto sobre la calidad de vida de las pacientes?.

Los resultados de este estudio, además de complementar la validez y confiabilidad publicado anteriormente ${ }^{10}$, son consistentes con los reportados en la versión original y portuguesa ${ }^{9,13}$. Esto quiere decir que, la versión evaluada en este estudio tiene la capacidad de detectar diferencias en la magnitud del constructo, que se conocen $a$ priori deberán ocurrir dado la eficacia conocida de una determinada intervención, en este caso el tratamiento médico o quirúrgico de la IU.

En el estudio previo de validación de la versión español-chileno del cuestionario ${ }^{10}$, se reportó un nivel alto de reproducibilidad para cada ítem del cuestionario, lo que sugiere que los cambios observados en el presente estudio son atribuibles al tratamiento y no al que se produce por la medición en dos ocasiones. Las pacientes con IU de esfuerzo fueron sometidas a un tratamiento quirúrgico efectivo ${ }^{15}$ y la versión español-chileno del cuestionario ICIQ-SF fue sensible para detectar este efecto, incluso después de controlar por la posible influencia del sobrepeso basal de estas mujeres. Por otro lado, el tratamiento médico de la IU fue específicamente en base a hormonas locales y/o anticolinérgicos ya que no se disponía del manejo kinésico institucional. Por esta razón y por el menor nivel de severidad basal, se esperaba resultados post-tratamiento de menor magnitud en estas pacientes; la versión español-chileno tuvo la capacidad de detectar estos cambios de menor magnitud, específicamente en el ítem de frecuencia y cantidad de pérdida de orina.

Dentro del proceso de validación de instrumentos, no existe un consenso sobre la forma de evaluar el criterio psicométrico de sensibilidad al cambio, habiendo una serie de propuestas con fortalezas y limitaciones ${ }^{12}$. En este estudio, se consideró la diferencia en el grado de severidad de la IU y la fuerte influencia del puntaje de severidad basal sobre el resultado final post-tratamiento a través de modelos estadísticos adecuados para medidas repetidas y la estructura de correlación entre ellas. Si bien no se fijó una medida de efecto clínicamente relevante (tamaño del efecto), lo que puede ser una limitación del estudio, si se pudo verificar a través de las pendientes de los coeficientes y el término de interacción en el modelo; es decir, que la magnitud del cambio detectada por el cuestionario fue menor en el tratamiento médico versus quirúrgico. Adicionalmente, al ser un método semi-paramétrico la distribución del puntaje y el tamaño de muestra no son relevantes al momento de hacer las comparaciones antes y después, sino más bien la correlación de los puntajes en cada paciente.

\section{Conclusión}

Los resultados de este estudio complementan la validez y confiabilidad de la versión español-chileno del cuestionario ICIQ-SF, confirmando la sensibilidad al cambio en una población de mujeres beneficiaras del Sistema Público de Salud. Esto quiere decir, que la versión evaluada tiene la capacidad de detectar cambios que ocurren como resultado de un tratamiento y puede ser usado en el ámbito clínico o de investigación.

\section{Referencias}

1. Hunskaar S, Arnold EP, Burgio K, Diokno AC, Herzog AR, Mallett VT. Epidemiology and Natural History of Urinary Incontinence. Int Urogynecol J 2000; 11: 3013019.

2. Holroyd-Leduc JM, Tannenbaum C, Thorpe KE, Straus SE. What type of urinary incontinence does this woman have? JAMA 2008; 299 (12): 1446-56. 
3. Thüroff JW, Abrams P, Andersson KE, Artibani W, Chapple CR, Drake MJ, et al. EAU Guidelines on Urinary Incontinence. European Urology 2011; 53 (3): 387-400.

4. Hermieu JF, Denys P, Fritel X. Étude comparée des recommandations publiées sur l'incontinence urinaire féminine. Progrès en urologie 2012; 22: 636-43.

5. Gormley A, Lightner DJ, Burgio KL, Chai TC, Clemens IQ, Culkin DJ, et al. Diagnosis and Treatment of overactive bladder (Non-Neurogenic) in adults: AUA/ SUFU guideline. The Journal of Urology 2012; 188 (6): 2455-63.

6. Gormley A, Lightner DJ, Farday M, Vasavada SP. Diagnosis and Treatment of Overactive Bladder (Non-Neurogenic) in Adults: AUA/SUFU Guideline Amendment. The Journal of Urology 2015; 193 (5): 1572-80.

7. Fritel X, Fauconnier A, Bader G, Cosson M, Debodinance P, Deffieux X, et al. Diagnosis and management of adult female stress urinary incontinence: guidelines for clinical practice from the French College of Gynaecologists and Obstetricians. Eur J Obstet Gynecol Reprod Biol 2010; 151: 14-9.

8. Valderas JM, Ferrer M, Alonso J. Instrumento de medida de calidad de vida relacionada con la salud y de otros resultados percibidos por los pacientes. Med Clin (Barc) 2005; 125 (Supl.1): 56-60.

9. Avery K, Donovan J, Peters TJ, Shaw C, Gotoh M, Abrams P. A brief and robust measure for evaluation the Symptoms and Impact of Urinary Incontinence. Neurourol Urodyn 2004; 23: 322-30.
10. Busquets M, Serra R. Validación del cuestionario International Consultation on Incontinence Questionnaire Short-Form (ICIQ-SF) en una población chilena usuaria del Fondo Nacional de Salud (FONASA). Rev Med Chile 2012; 140: 340-6.

11. Husted JA, Cook RJ, Farewell VT, Gladman DD. Methods for assessing responsiveness: a critical review and recommendations. J Clin Epidemiol 2000; 53 (5): 45968.

12. Espuña Pons M, Puig Clota M. Síntomas del tracto urinario inferior en la mujer y afectación de la calidad de vida. Resultados de la aplicación del King's Health Questionnaire. Actas Urol Esp 2006; 30 (7): 684-91.

13. Tamanini J, Dambros M, D’Ancona C, Palma P, Rodrigues-Netto Jr N. Responsiveness to the Portuguese version of the international consultation on incontinence questionnaire-short form (ICIQ-SF) after stress urinary incontinence surgery. Int Braz J Urol 2005; 31(5): 482 90.

14. Tan PF, Yang LL, Ou RB, Tang P, Yang WJ, Huang $\mathrm{JB}$, et al. Effectiveness and complication rates of tension-free vaginal tape, transobturator tape, and tension-free vaginal tape-obturator in the treatment of female stress urinary incontinence in a medium- to long-term follow up. Meta-analysis of randomized controlled trials. Saudi Med J 2014; 35 (1): 20-32.

15. García M, Rodríguez F, Carmona L. Sensibilidad al cambio de las medidas de desenlace. Reumatol Clin 2008; 4 (6): 240-7. 